\title{
Epiduroscopic intervention in patients with a failed back surgery syndrome
}

\author{
Griger $\mathrm{M}^{1}$, Kocan $\mathrm{L}^{2}$, Rapcan $\mathrm{R}^{1,3,4}$, Matias $\mathrm{M}^{5}$, Burianek $\mathrm{M}^{3}$, Kocanova $\mathrm{H}^{6}$, Rapcanova $\mathrm{S}^{3}$, \\ Mlaka $\mathrm{J}^{7}$, Zahorec $\mathrm{R}^{8}$, Vaskova $\mathrm{J}^{9}$ \\ Department of Medical and Clinical Biochemistry, Faculty of Medicine, Pavol Jozef Safarik University, \\ Kosice, Slovakia. janka.vaskova@upjs.sk
}

\begin{abstract}
BACKGROUND: Epiduroscopy is a percutaneous endoscopic technique with an interesting diagnostic and therapeutic potential.

AIM: The study was aimed to evaluate the effectiveness of this modern minimally invasive method in treating pain together with the accompanying neurological symptoms of patients with a failed back surgery syndrome (FBSS).

RESULTS: In the retrospective multicentre clinical study, we monitored the effect of epiduroscopic performance in 40 patients with FBSS before epiduroscopy and after 6 and 12 months. The results of the study indicated a significant improvement in back pain after 6 months, at $p<0.05$. At the same time, a significant improvement was observed in pain radiating to the lower limbs after 6 and 12 months at $p<0.05$, as well as a significant improvement in the Oswestry Disability Index of the limbs after 6 months and after 12 months $(p<0.05)$. CONCLUSION: The current summarised results indicate a significant positive effect of epiduroscopy in the treatment of chronic back pain with a reduction in radiating pain to the lower extremities and an increase in patients' quality of life after the procedure (Tab. 2, Fig. 1, Ref. 23). Text in PDF www.elis.sk KEY WORDS: epiduroscopy, failed back surgery syndrome, Oswestry Disability Index, pain, quality of life.
\end{abstract}

\section{Introduction}

In spinal surgery, a clinical condition called post-laminectomy syndrome is often encountered. In Anglo-Saxon literature this is also referred to as a failed back surgery syndrome (FBSS), or spinal surgery failure syndrome. This syndrome manifests after one or more operations on the back in the same or adjacent intervertebral spaces, most often in the lumbar and sacral spine, and the incidence increases with the number of operations $(1,2)$.

The syndrome manifests as the onset or persistence of chronic back pain, often with pain radiating to lower limbs. The patho-

${ }^{1}$ Europainclinics, Bratislava - Nové mesto, Slovakia, ${ }^{2}$ Clinic of Anaesthesiology and Intensive Care Medicine, East Slovak Institute of Cardiovascular Disease, Košice, Slovakia, ${ }^{3}$ Europainclinics, Prague, Czech Republic, ${ }^{4} \mathrm{Eu}-$ ropainclinics, Bardejov, Slovakia, ${ }^{5}$ Europainclinics, Brno-Starý Lískovec, Czech Republic, ${ }^{6} \mathrm{Clinic}$ of Anaesthesiology and Intensive Care Medicine, Railway Hospital and Clinic Košice, Košice, Slovakia, ${ }^{7}$ Europainclinics, Poliklinika Terasa, Košice, Slovakia, ${ }^{8}$ 2nd Department of Anestesiology and Intensive Medicine, Faculty of Medicine, Comenius University and St. Elizabeth Cancer Institute, Bratislava, Slovakia, and ${ }^{9}$ Department of Medical and Clinical Biochemistry, Faculty of Medicine, Pavol Jozef Šafárik University, Košice, Slovakia

Address for correspondence: J. Vašková, Dr, PhD, Department of Medical and Clinical Biochemistry, Faculty of Medicine, Pavol Jozef Šafárik University in Košice, Trieda SNP 1, SK-040 66 Košice, Slovakia.

Phone: +421.552343232 physiology of FBSS is complex, with mixed neuropathic and nociceptive elements (e.g., mechanical and inflammatory) and activation of the sympathetic nervous system (3). In this context, it is important to mention the myofascial pain that arises in the paraspinal muscles the result of their denervation and atrophy during dissection and prolonged retraction. These intraoperative changes are further potentiated by postural changes in the postoperative period. Following a loss of lumbar lordosis due to fixation, paraspinal muscles can progress to chronic spasms and subsequent atrophy. Typically, the patient compensates this situation by hyperextension of the thoracolumbar spine, which further exacerbates poor posture and thus pain in the long term. This myofascial pain is also referred to as "fusion disease" (4).

Epidural and perineural fibrosis also plays an important role. Epidural fibrosis and the formation of adhesions in the epidural space is, in principle, a normal response to surgery and occurs to some extent in most patients after such an operation. While the exact significance of this fibrosis has not been clearly established in the development of FBSS, many authors consider it to be one of the main causes (5). The practical classification of the causes of FBSS is based on preoperative, operative and postoperative factors (6), with diagnosis based mainly on the patient's medical history and clinical examination. Imaging techniques such as computed tomography and magnetic resonance imaging help to identify some factors that still generate pain in the postoperative period (persistent foraminal stenosis, pseudoarthrosis, instability). 
In most cases, however, they are unable to detect the presence of epidural fibrosis or epidural adhesions (7).

Treatment is complex, with the patient usually visiting several specialists, including a neurosurgeon, pain specialist, rehabilitation doctor, and an orthopaedist. In most treatment cases, a conservative approach is chosen, based on combination complex therapy, which includes pharmacotherapy aimed at treating neuropathic pain, repeated applications of corticoids into the epidural space, and a wide range of neuromodulation and rehabilitation techniques. However, despite intensive efforts, these therapies often fail, resulting in a persistent chronic pain and a reduced quality of life. In selected indicated cases, spinal cord stimulator implantation is possible. This is a costly treatment and is not suitable for every patient with this syndrome; indeed, the syndrome must meet relatively strict criteria (8).

In recent years, a minimally invasive endoscopic technique has emerged, namely epiduroscopy. This can be included as a precursor in the SCS implantation algorithm and has come to the forefront of diagnostic and therapeutic options for patients with FBSS. During the epiduroscopic procedure, a flexible endoscope is inserted into the epidural space, which enables visual assessment and possible treatment of pathological processes in patients with suspected epidural pathology. Epiduroscopy is proving to be a more sensitive method in the detection of epidural pathology compared to magnetic resonance imaging (MRI), especially due to the functional nature of the examination (7). This is especially true, when it is possible to test the individual structures, thereby helping to determine whether their mechanical irritation causes concordant pain, thus representing a pain generator in given situation.

The anterior epidural space is narrower and more difficult to access for the epiduroscope, which may increase the risk of perforation of the dura. There are, however, structures present that contribute to root pain. When manipulated in the anterior epidural space, concordant pain is often caused by touching the pathological structure, which may indicate a source of pain. As the posterior epidural space is larger and more easily accessible, most instruments end up in this space without a targeted navigation. It can be assumed that most postoperative fibrous changes are localised at this site, but it is rarely possible to induce concordant pain by touching anatomical-pathological structures with the instruments. There are no structures involved in radicular pain in the posterior epidural space. However, in our experience, there is a group of patients, who feel the benefit only after treatment of the posterior epidural space, this is probably due to the complex nature of the effects of epiduroscopy (9). The mechanism of the therapeutic effect of epiduroscopy is mainly through the targeted disruption of epidural fibrotic adhesions and mechanical release of mutually adhered individual anatomical structures, so-called adhesiolysis, as well as rinsing the epidural space with saline, thereby reducing inflammatory mediators at the site of pathology and inflammation. Another significant effect is the application of drugs that have an anti-inflammatory effect (10).

The aim of this study was to evaluate the effectiveness of epiduroscopic procedures after 6 and 12 months on the reduction of pain in the lower back and pain radiating to the lower limbs in patients with FBSS.

\section{Materials and methods}

In the retrospective analysis from January 2016 to January 2019, patients treated for chronic pain were followed, both at the clinic of the East Slovak Institute of Cardiovascular Diseases in Košice and at the EuroPainClinics algesiology clinics in Košice, Bratislava and Bardejov. Only patients, who met the following indication criteria could be included in the study: Patients with chronic pain and FBSS after one or repeated unsuccessful spinal surgeries, who also underwent epiduroscopic surgery. All the patients had to be injected periprocedurally into the epidural space with the injectable corticosteroid methylprednisolone at a dose of $80 \mathrm{mg}$. The age of patients enrolled in the study ranged from 35 to 70 years.

The study was approved by the ethics committee of VÚSCH a.s. in Košice under the registration number $1 / 2019$ VUSCH and was registered in the international database Clinicaltrials.gov with assigned identification number: NCT03916666. Patients were informed by phone about possible participation in the clinical study, while consent to participate in the study and processing of personal data were requested in person.

Participants were assigned a 7-digit identification number (ID) and then an electronic SURVEY-Google Forms questionnaire was sent to each participant's email address, with questions focused on three clinical periods: T1 before epiduroscopy, T2 6 months after epiduroscopy, and T3 12 months after epiduroscopic surgery. The following parameters were monitored: Oswestry Disability Index, numerical scale of pain radiating to the lower limbs, numerical scale of lower back pain, numerical scale of quality of life (EQ VAS) and consumption of analgesics.

Summarised data of participant age, gender, ASA, BMI, spread of dermatome pain and type of mechanical therapeutic intervention were obtained from preoperative algesiological examination records and surgical protocols from the EuroPainClinics database (see patient characteristics table). Statistical analysis of electronically collected data was performed at the Department of Medical and Clinical Biochemistry, Faculty of Medicine, Pavol Jozef Šafárik University in Košice.

\section{Technical performance}

The epiduroscopic procedure was performed under aseptic conditions in the operating room under a local anaesthesia and in mild analgesia monitored by an anaesthesiologist, to ensure cooperation. There was also the option of using protective artificial lung ventilation (11). This cooperation is crucial and avoids some potentially serious complications. In addition, the patient's ability to communicate with the performing physician during the procedure adds a dynamic dimension to an otherwise static examination method and facilitates the identification of the pain generator.

Access to the epidural space is gained in most cases via the hiatus sacralis through puncture and dilation according to Seldinger or direct access with dilatation by the pean instrument. In 
the case of anatomical peculiarities (bone stenosis of the sacral canal, coccyx deformities, large Tarlow's cyst), there is another possible route of access through the foramen S1 $(12,13)$. After insertion of the epiduroscope into the epidural space, the site of the presumed pathology is identified under intermittent sciascopic and direct visual inspection. A disposable navigable epiduroscope with an outer diameter of about $3.4 \mathrm{~mm}$ with several working channels (a channel for inserting flexible $1 \mathrm{~mm}$ optics with a light source, a channel for instruments used for adhesiolysis and ports for flushing targeted drug delivery) was also used for the procedure. Other special instruments were also used in the form of expansion balloons, optical laser fibres, and graspers. The most frequently used tool in our conditions was the QMR probe (Resaflex), which enabled mechanical adhesiolysis and evaporation of tissues by the method of molecular resonance at relatively low temperatures. During the inspection of the epidural space, we specifically looked for pathological changes, especially evaluating fibrous tissue, hyperaemia deposits, pathological vasculature, and the absence of adipose tissue.

All attempts were made to perform the procedure in the presumed place of pathology. According to the clinical picture, focus was given to structures, whose irritation could cause a concordant pain. After reaching the site of pathological inflammatory changes, adhesiolysis was performed with the blunt tip of an epiduroscope, with a QMR probe, or with subsequent targeted application of drugs (such as corticoids and/or the enzyme hyaluronidase).

\section{Monitored parameters}

The Numerical Pain Scale (VAS) is an internationally recognised tool for determining pain intensity. The patient determines the intensity of pain on an 11-point scale (0-10), where 0 means no pain and 10 means the most severe pain the patient can imagine.

The Oswestry Disability Index is derived from the "Oswestry low back pain" questionnaire, which quantifies a disability caused by lower back pain. The questionnaire includes 10 questions related to pain intensity, lifting, levels of self-care, ability to walk, sit, sex life, ability to stand, social life, quality of sleep and ability to travel. Each categorical question has 6 optional answers, describing different alternatives. The answers are ranked from the most favourable variant to the most unfavourable possible.

This scale records the current overall feeling of health on a vertical visual analogue scale, where the endpoints are labelled "Best Feeling Health You Can Imagine" and "Worst Feeling Health You Can Imagine". EQ VAS represents a quantitative measure of a patient's perception of their overall health.

\section{Statistical analysis}

Methods of descriptive statistics (percentage values, arithmetic mean, minimum, maximum, median standard deviation, and frequencies) were used to evaluate the obtained data. The normality of the distribution of values was verified by the Shapiro-Wilk test and the homogeneity of the variances by the Levene test. Differences between dependent continuous values were analysed by the Kruskal-Wallis test. Differences in continuous independent variables were analysed by a two-sample t-test with equal vari- ance. The results of the differences, when comparing the groups with the above tests, were marked as statistically significant at a significance level of $\mathrm{p}<0.05$. Statistical analyses were processed in MS Excel and the statistical software SPSS 11.0.

\section{Results}

The study evaluated 80 patients, whose basic descriptive characteristics are summarised in the Table 1. Patients had the mean age of 54 years, with slightly more men than women. The most common spread of pain was in dermatomes L4-L5, then L5-S1 and S1. Almost a third of patients had previously undergone mechanical radiofrequency therapy and 10 patients had dysfunction or paralysis of the bladder.

The monitored parameters were recorded in three periods before the procedure, after 6 months and after 12 months. The evaluation of the disability assessment by lower back pain showed that there was a significant improvement in patients with FBSS after epiduroscopic surgery, not only after 6 months, but also after 12 months at $\mathrm{p}<0.05$ (Tab. 2, Fig. 1). Regarding the differentiation of pain intensity according to the VAS assessment, patients improved after 6 months; however, the perception of a significant improvement in pain of the lower extremities persisted in patients after both 6 and 12 months. Despite this, the assessment of the overall improvement in health according to the EQ VAS questionnaire did not lead to a significant improvement after 6 or 12 months (Tab. 2, Fig. 1).

\section{Discussion}

FBSS affects a relatively high percentage of operated patients, with available literature figures between 10-40\% (14). The suc-

Tab. 1. Descriptive characteristics of the group of examined patients.

\begin{tabular}{lr}
\hline Characteristics of patients & \\
\hline Number of patients before the procedure & 80 \\
Number of patients after 6 months & 80 \\
Number of patients after 12 months & 80 \\
Age (years) & $(35-70)$ \\
Gender (M/F) & $42 / 38$ \\
ASA & $(17-25) 21$ \\
BMI & 2 \\
\hline Spread of pain in dermatomes before procedure & 2 \\
L2 & 3 \\
L3-L4 & 27 \\
L4-L5 & 15 \\
L5 & 16 \\
L5-1 & 16 \\
S1 & \\
\hline Mechanical therapeutic intervention & 10 \\
Balloon decompression & 9 \\
Laser & 31 \\
Radiofrequency & 10 \\
\hline Complications & 1 \\
Paralysis of the bladder & 8 \\
Neurological deficit in dermatomes & 0 \\
Dura mater punction & \\
Infection, bleeding &
\end{tabular}


Tab. 2. Evaluation of pain intensity in the lower back, lower limbs according to the VAS and Oswestry disability index questionnaires as well as the feeling of general health (EQ VAS) in patients before surgery and 6- and 12-months after epiduroscopic surgery.

\begin{tabular}{|c|c|c|c|c|c|c|}
\hline \multirow{2}{*}{ Parameter } & \multicolumn{2}{|c|}{ before procedure } & \multicolumn{2}{|c|}{6 months follow-up } & \multirow[b]{2}{*}{ CI $95 \%$} & \multirow[b]{2}{*}{$\mathrm{p}$} \\
\hline & Median & SD & Median & SD & & \\
\hline Oswestry Disability Index & 50 & 17.203 & 41 & 19.244 & $3.883-15.357$ & $0.010 *$ \\
\hline VAS back & 7 & 20.579 & 5 & 2.503 & $0.568-2.168$ & $0.001 *$ \\
\hline VAS lower limbs & 7 & 10.596 & 5 & 2.207 & $1.040-2.207$ & $0.001 *$ \\
\hline \multirow[t]{3}{*}{ EQ VAS } & 37.5 & 16.954 & 45.00 & 14.855 & $-0.984-0.211$ & 0.060 \\
\hline & \multicolumn{2}{|c|}{ before procedure } & \multicolumn{2}{|c|}{12 months follow-up } & & \\
\hline & Median & $\mathrm{SD}$ & Median & SD & CI 95\% & $\mathrm{p}$ \\
\hline Oswestry Disability Index & 50 & 17.203 & 41 & 18.654 & $-0117-11.417$ & $0.046^{*}$ \\
\hline VAS back & 7 & 2.579 & 6.5 & 2.451 & $-0.261-1.561$ & 0.058 \\
\hline VAS lower limbs & 7 & 1.596 & 6 & 1.161 & $0.0776-1.161$ & $0.025 *$ \\
\hline EQ VAS & 37.5 & 16.954 & 44.5 & 14.855 & $-5.881-4.590$ & 0.808 \\
\hline
\end{tabular}
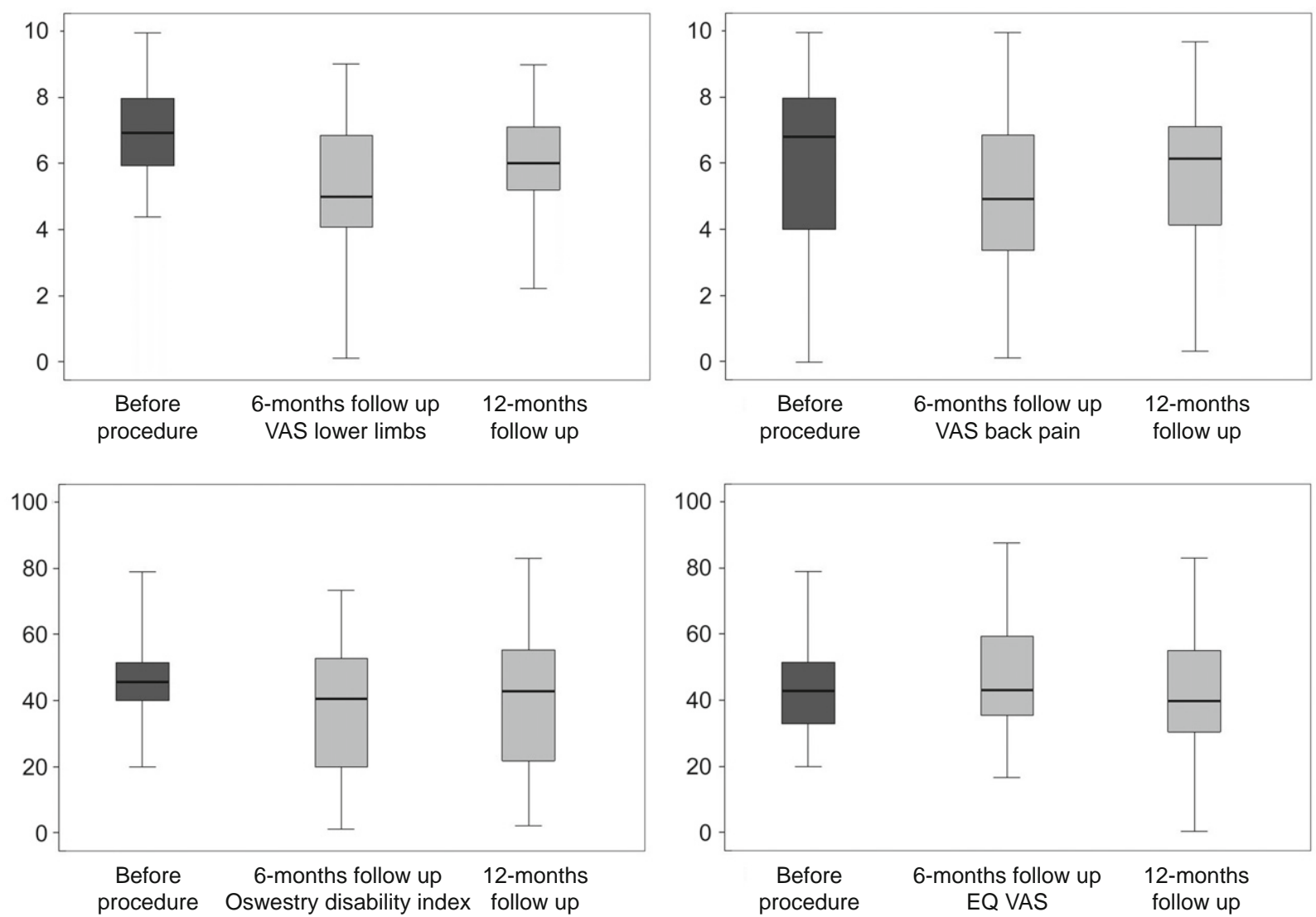

Fig. 1. Evaluation of patients' scores on the Numerical Pain Scale (VAS) for lower limbs, back pain, Oswestry Disability Index and overall health (EQ VAS) before treatment, 6 months, and 12 months after treatment.

cess of the operation decreases with the number of repetitions: in the first, $>50 \%$; in the second, $30 \%$; in the third, $15 \%$; and in the fourth, $<5 \%$ (15). As the number of recurrent operations increases, so does the incidence of back surgery syndrome (1). The causes of FBSS are both the failure to achieve the goal of surgery, postoperative changes causally related to surgery and the psychological profile of the patient. Failure to reach the target means that pathological changes are still identifiable even after surgery; one study with 170 FBSS patients identified foraminal stenosis in $29 \%$ of patients, discogenic pain in $17 \%$, pseudoarthrosis in $14 \%$, neuropathic pain in $9 \%$, instability in $5 \%$, and psychological problems in $3 \%$ (16).

Many authors consider epidural and perineural fibrosis to be important in the development of FBSS-related pain. This can occur after any open operation that requires manipulation of the epidural space. Surgical intervention is most likely to trigger a cascade of local inflammatory changes in the epidural space that form a part of the natural healing process after open manipula- 
tion, and in the final phase of the process, fibrous tissue is formed. These secondary fibrous structures are actually a secondary form of healing, namely the formation of postoperative scar tissue. The degree of scarring of the epidural space depends partially on the extent of the operation. It can now be said with certainty that it is also dependent to a large extent on the genetic predisposition of the operated individual (17).

More severe epidural fibrosis occurs in $5-30 \%$ of cases after laminectomy without fusion $(18,19)$. Two basic mechanisms, the fibrosis scar pressure and inflammatory mediators, are pathophysiologically responsible for the generation of pain in epidural fibrosis. Together, they are responsible for changes in the vascular supply of the nerve and myelin sheaths. Blood accumulation in the epidural space further exacerbates scar formation (20). The diagnosis of epidural fibrosis is routinely made on the basis of conventional imaging methods such as CT and MRI, but these have been shown not to detect its presence with any reliability. In one study comparing the detection capability of MRI and epiduroscopy, MRI detected fibrosis in $16 \%$ of the patients with FBSS, while epiduroscopy detected more than $90 \%$ (7).

FBSS is a typical example of chronic pain and is a major problem for patients and their surroundings. Clinical signs include somatic changes, decreased mobility, muscle atrophy, decreased pain threshold, imbalance of the autonomic system, adversely affecting other functions of organ systems such as: the cardiovascular, gastrointestinal, urinary and others. A common side effect encountered in patients with FBSS is varying degrees of mood disorder, especially in the form of anxiety-depressive disorder, which worsens the experience of chronic pain. The patient's social activity is significantly disrupted, which limits social, work and intimate life. FBSS negatively affects an individual's economic self-sufficiency $(21,22)$. In general, the syndrome significantly reduces various aspects of a patient's quality of life (23).

In the retrospective analysis, attention was given to the possible benefit of epiduroscopic performance in the observed group of patients, where a significant reduction in lower back pain was recorded after 6 months and a significant reduction in pain radiating to the lower limbs lasted after 6 and 12 months.

Determination of a patient's quality of life, as measured by changes in the Oswestry Disability Index and aimed at assessing individual daily activities limited by the clinical condition in chronic back and/or lower limb pain, also indicates a significant improvement in the patient's condition after 6 and 12 months. Paradoxically, patients assessed their condition on an analogous quality of life scale without a significant change after endoscopic surgery. This phenomenon could be explained by the fact that chronic pain in FBSS is multifactorial, complex and difficult to treat. It is very often associated with a significant depressive component, which further negatively affects the patient's perception of quality of life. These psychological factors are often present before surgery and some authors consider them to be a comorbidity (Förster et al, 2013), which further worsens the clinical outcome of the operation and subsequent treatment. Despite the pain relief described above, certain limitations persist. The average value of lower back pain before surgery was VAS 7, after 6 months it decreased to VAS 5 and after 12 months it slightly increased to VAS 6.5. A slightly better result was found for pain radiating to the lower limbs, where the initial mean value before surgery was VAS 7, after 6 months it decreased to VAS 5, and after 12 months it slightly increased to VAS 6. However, the resulting values of persistent pain after 6 and 12 months were still considered to be moderate pain, which means that the patient is dependent on pharmacotherapeutic procedures.

In the group monitored in this study, the occurrence of some perioperative complications were recorded from the archived documentation, which are further monitored within the postoperative therapeutic management regime.

A relatively common complication is short-term (3-6 hours) urinary retention, associated with motor paralysis of the lower limbs. Complications of bladder paralysis occurred in 10 patients according to documented records, and short-term neurological deficit in the dermatome was reported in one case. All patients had a total resolution of symptoms within 6 hours. These complications can be explained by the possible penetration of the local anaesthetic intrathecally, through the mechanically damaged dural sac. The local anaesthetic, which penetrates the cerebrospinal fluid, brings about the onset of spinal anaesthesia, which manifests as temporary motor paralysis and urinary retention. After the effect of the local anaesthetic subsides, paralysis and urinary retention disappear as well as the neurological deficit in the dermatome. Due to the use of mechanical instruments in the epidural space, the risk of dural puncture cannot be ruled out. This complication has been reported perioperatively in 8 cases. None of the patients experienced post-puncture headaches or other clinical symptoms that would require a subsequent treatment. The risk of epidural haemorrhage and epidural abscess should also be mentioned, when evaluating possible complications associated with epiduroscopy. These risks are minimal, when performing the procedure correctly, as there is a direct optical control over possible bleeding and immediate possibility of coagulation under optical control. The risk of introducing infection is also minimized by the use of disposable, sterile instruments and strict adherence to the principles of surgical asepsis and antisepsis.

There were no cases of infection or epidural haemorrhage nor of infection or epidural haemorrhage in our study population.

In order to optimise the therapeutic process of pain treatment in the patient it is very important not to forget the precise diagnosis of other pain generators such as: intervertebral articulation, unoperated intervertebral discs, or a further diagnosis of movement stereotypes associated with myofascial pain progression of spinal structure disease.

\section{Conclusion}

Epiduroscopy, as one of the more advanced minimally invasive spinal techniques, is primarily important as a complementary diagnostic method that determines the state of pathological involvement of the epidural space. It is possible that optical visualisation reveals pathological changes that are undetectable or very difficult to detect on MRI. At the same time, it has the potential for therapeutic intervention, both in complicated patients after 
$727-732$

spinal surgery, where other therapeutic options fail, as well as in patients without a previous back surgery. As a minimally invasive endoscopic technique, it carries less potential for complications.

The available evidence from multicentre randomised studies supports the effectiveness and safety of epiduroscopy, and therefore deserves a stable place in the treatment of specific types of spinal pathology. Epiduroscopy is currently a well-established treatment method in Slovakia as well as in the Czech Republic.

\section{References}

1. Pawl R. Lumbar spinal surgery, where have we gone? Surg Neurol 2008; 69 (6): 558-560.

2. Kozák J. Neuromodulace v léčbě chronické bolesti. Sanquis 2005; 41 (2): 21 .

3. Van Buyten JP, Linderoth B. The failed back surgery syndrome: Definition and therapeutic algorithms - An update. Eur J Pain Suppl 2010; 4 (4): 273-286.

4. Onesti S. Failed back syndrome. Neurologist 2004; 10 (5): 259-264.

5. Bosscher H, Heavner J. Diagnosis of the vertabral level from which low back or leg pain originates. A comparison of clinical evaluation. Pain Pract 2012; 12 (7): 506-512.

6. Chan Ch, Peng P. Failed back surgery syndrome. Pain Med 2011; 12 (4): 577-606.

7. Bosscher HA, Heavner JE. Incidence and Severity of Epidural Fibrosis after Back Surgery: An Endoscopic Study. Pain Pract 2010; 10 (1): 18-24.

8. Rapcan R, Mlaka J, Venglarcik M, Vinklerova V, Gajdos M, Illes R. High-frequency-spinal cord stimulation. Bratisl Lek Listy 2015; 116 (6): 354-356.

9. Griger M, Mláka J. Technické aspekty epiduroskopie. Bolest 2017; 20 (2): 89-96.

10. Rapčan R, Kočan L, Mláka J, Burianek M, Kočanová H, Rapčanová S, Hess M, Hammond A, Griger M, Venglarčík M, Gajdoš M, Vašková J. A randomized, multicenter, double-blind, parallel pilot study assessing the effect of mechanical adhesiolysis vs adhesiolysis with corticosteroid and hyaluronidase administration into the epidural space during epiduroscopy. Pain Med 2018; 19 (7): 1436-1444.
11. Čandík R, Rybár D, Sabol F, Kolesár A, Saladiak S, Imrecze Š, Beňová J, Galková K. Vplyv parametrov umelej ventilácie plúc počas anestézie na mechanickú energiu pohltenú plúcami. Anestéziol Intenzívna Med 2016; 5 (2): 67-70.

12. Avellanal M, Diaz-Reganon G. Interlaminar approach for epiduroscopy in patients with failed back surgery syndrome. Br J Anaesth 2008; 101 (2): 244-249.

13. Avellanal M, Diaz-Reganon G, Orts A, Gonzalez-Montero L, Riguelme I. Transforaminal epiduroscopy in patients with failed back surgery syndrome. Pain Physician 2019; 22 (1): 89-95.

14. Thomson S, Jacques L. Demographic characteristics of patients with severe neuropathic pain secondary to failed back surgery syndrome. Pain Pract 2009; 9 (3): 206-215.

15. Nachemson AL. Evaluation of results in lumbar spine surgery. Acta Orthop Scand Suppl 1993; 251: 130-133.

16. Waguespack A, Schofferman J, Slosar P, Reynolds J. Etiology of long-term failures of lumbar spine surgery. Pain Med 2002; 3 (1): 18-22.

17. Geisler FH. Prevention of epidural fibrosis: current methodologies. Neurol Res 1999; 21 (Suppl 1): S9-22.

18. Trescot AM, Chopra P, Abdi S, Datta S, Schultz DM. Systematic review of effectiveness and complications of adhesiolysis in the management of chronic spinal pain: an update. Pain Physician 2007; 10 (1): 129-146.

19. Jönsson B, Strömqvist B. Repeat decompression of lumbar nerve roots. A prospective two-year evaluation. J Bone Joint Surg Br 1993; 75 (6): 894-897.

20. Häckel M, Masopust V, Bojar M, Ghaly Y, Horínek D. The epidural steroids in the prevention of epidural fibrosis: MRI and clinical findings. Neuro Endocrinol Lett 2009; 30 (1): 51-55.

21. Martuliak I. Nenádorová bolest' v algeziologickej praxi-vybrané aspekty. Paliatívna medicína a liečba bolesti 2008; 1 (1): 8-11.

22. Martuliak I. Možnosti liečby bolesti chrbta -2 . čast'. Via pract 2009; 6 (3): 124-126.

23. Rapčan R, Kočan L, Witkovsky V, Mláka J, Griger M, Burianek M, Rapčanová S, Hammond A, Poliak L, Tirpák R, Šimonová J, Sabol F, Vašková J. EQ-5D-5L questionnaire as suitable assessment of quality of life after epiduroscopy : Multicenter randomized double-blind pilot study. Wien Klin Wschr 2020. DOI: 10.1007/s00508-019-01590-z.

Received May 14, 2020. Accepted May 22, 2020. 